GANIT J. Bangladesh Math. Soc. (ISSN 1606-3694) 38 (2018) 1-10

DOI: https://doi.org/10.3329/ganit.v38i0.39781

\title{
NUMERICAL BIFURCATION ANALYSIS TO STUDY PERIODIC TRAVELING WAVE SOLUTIONS IN A MODEL OF YOUNG MUSSEL BEDS
}

\author{
Md. Ariful Islam Arif* and M. Osman Gani \\ Department of Mathematics, Jahangirnager University, Savar, Dhaka-1342, Bangladesh. \\ Corresponding author, E-mail:* md.arifulislamarif@yahoo.com
}

Received: 10-09-2017 Accepted on: 25-11-2018

\begin{abstract}
Self-bottomed mussel beds are dominant feature of ecosystem-scale self-organization. Regular spatial patterns of mussel beds in inter-tidal zone are typical, aligned perpendicular to the average incoming tidal flow. In this paper, we consider a two-variable partial differential equations model of young mussel beds. Our aim is to study the existence and stability of periodic traveling waves in a one-parameter family of solutions. We consider a parameter regime to show pattern existence in the model of young mussel beds. In addition, it is found that the periodic traveling waves changes their stability by two ways: Hopf type and Eckhaus type. We explain this stability by the calculation of essential spectra at different grid points in the two-dimensional parameter plane.
\end{abstract}

Keywords: Essential spectrum, Hopfbifurcation, Eckhaus bifurcation, mussel beds, periodic traveling wave, reaction-diffusion-advection.

\section{Introduction}

Banded spatial patterns of mussel beds are a striking characteristic feature within spatial ecology [1], [2], [8], [10], [13], [18], [19]. Different types of landscape-scale patterns, e.g., arid ecosystems [4], savannahs [7], [14], tidal freshwater marshes [5], intertidal mudflats [21], mussel beds [6], have documented in field data. Subsequently, Mussel beds have been found in many parts of the earth, e.g., Waddensea [3], MenaiStriat (UK) [6], North sea on the coastline of Denmark, Germany and Netherlands [16]. A soft-bottomed system means that the mussels adhere only to one another or to debris such as shell fragments. Banded (striped) patterns of mussel beds typically are aligned perpendicular to the average incoming tidal flow, with a wavelength of several meters. Spatial patterns in mussel beds distinctly develop at two different scales such as large-scale banded patterns and small-scale net-shaped patterns. In the water column at large spatial scales, mussels compete for algae as the high flow rate of the tidal water but expedite each other at fewer scales by regionally growing sediment. This exchange between facilitation and competition reveals the striped patterns. At the smallest scale, each mussel aggregate by average density dependent movement and they actively move into small clusters to form a reticulated network of clusters. As well as, mussels connect to each other by average byssal threads to protect from predation and 
dislodgement. Therefore, the large-scale banded patterns and small-scale clusters occur from a self-organization process, meaning that the patterns appear from the interplays between the mussels. Pattern formation at the each level is very quick while the large-scale banded patterns need several periods to grow. Mussel beds afford a novel possibility to study how pattern formation at distinct spatiotemporal scales changes the functioning of spatial ecosystems.

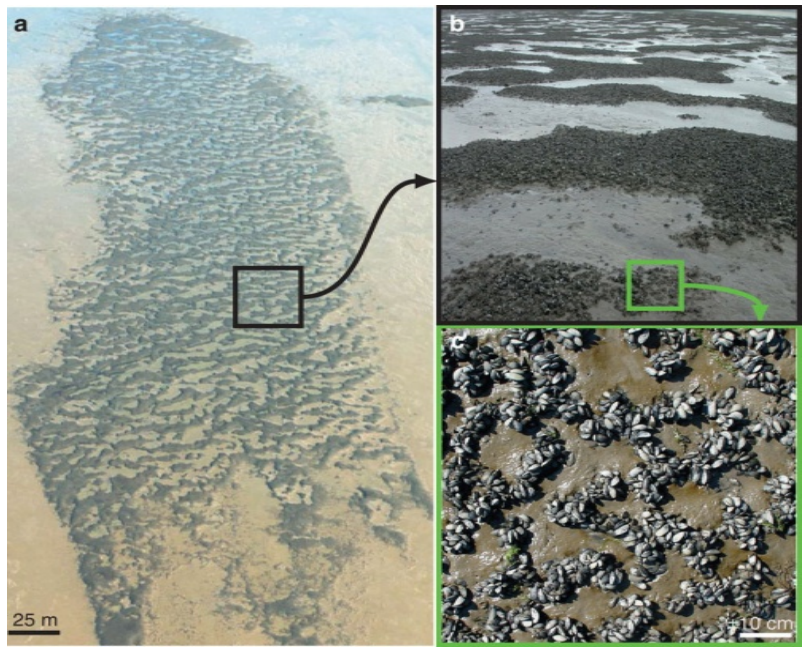

Fig. 1. Landscapes of mussel bed on an inter-tidal flat represent self-organized spatial patterns [9].

A wide variety of theoretical models [20] is playing an important role in understanding the ecological mechanisms responsible for these various patterns since the opportunities for empirical study are typically very limited. Using mathematical modeling, one can predict how spatial patterns might be affected by varies in environmental parameters. Van de Koppel [6] proposed a mathematical model based on "reduced losses" hypothesis. The "reduced losses" hypothesis for banded patterns in soft-bottomed mussel beds is a scale difference in facilitation between neighboring mussels and competition among the mussels for algal food sources.

In this paper, we provide a systematic analysis of the model of mussel band formation. This analysis may provide a putative explanation for the widespread occurrence of banded patterns in mussels beds and other striped ecosystems. Thus, our aim is to study the existence of periodic traveling wave solutions (PTWs) in the model (1). We establish the existence of the PTWs of the model in a two-dimensional parameter plane. We also show two types of unstable PTWs in the model in the same set-up.

The remainder of the paper is organized as follows. In Section 2, we give a brief overview of the model for mussel beds. In Section 3, we discuss the existence of PTWs in the model. Our work in Section 4 presents stability of PTWs. Finally, we discuss the mathematical and ecological implications of our results in Section 5. 


\section{Model}

The hypothesis of van de Koppel [6] has been formulated in terms of algal concentration $a(x, t)$ in the water layer overlying the mussel bed and mussel biomass $m(x, t)$ on the sediment. The equations have the form

$$
\begin{aligned}
& \frac{\partial a}{\partial t}=\overbrace{\alpha(1-a)}^{\begin{array}{c}
\text { transferto } \\
\text { fromwaterlayer }
\end{array}}-\overbrace{a m}^{\begin{array}{c}
\text { consumptiom } \\
\text { bywater }
\end{array}}+\overbrace{\beta \frac{\partial a}{\partial x}}^{\begin{array}{c}
\text { advection } \\
\text { bytide }
\end{array}}, \\
& \frac{\partial m}{\partial t}=\underbrace{\delta a m}_{\text {birth }}-\underbrace{\frac{\gamma m}{(1+m)}}_{\begin{array}{c}
\text { dislodgement } \\
\text { by water }
\end{array}}+\underbrace{d_{w} \frac{\partial^{2} m}{\partial x^{2}}}_{\begin{array}{c}
\text { random } \\
\text { movement }
\end{array}},
\end{aligned}
$$

where the parameter is in dimensionless form. Here, spatial coordinatexis measured with distance from the shore andtrepresents time. The positive constant $\beta$ describes the water flow rate. The parameter $\alpha$ reflects the renewal rate of algae in the water layers. The parameter $\delta$ scales the potential growth of algal in the upper water layers. The term $\gamma$ represents mussel maximum mortality. The termrepresents advection by tidal current which is multiplied by the constant.

An important preamble to the investigation of patterns is to find the homogeneous steady state. For all parameter values, systems (1) has a stable trivial steady state $a=1, m=0$, corresponding to the existence for algae and absence of mussels. When $\delta>\gamma>\delta \alpha$ there is a non-trivial homogeneous steady state

$$
a=\frac{\gamma-\delta \alpha}{\delta \alpha}, m=\frac{\alpha(\delta-\gamma)}{\gamma-\delta \alpha} .
$$

General conditions on the stability of this steady state are parameter dependent. As well as, this non-trivial steady state is unstable to spatially inhomogeneous perturbations and stable to homogeneous perturbations for some parameter values.

\section{Existence of Traveling Wave Solutions}

The spatial patterns can be investigated via the traveling wave ordinary differential equations satisfied by a traveling wave coordinate $z=x-c t$, where $x$ and $t$ are the space and time coordinates respectively, and $c$ denotes the wave speed. Now, substituting $a(x, t)=A(z)$ and $m(x, t)=M(z)$ in (1), we obtain four-dimensional ordinary differential equation (ODE) system given as follows:

$$
\frac{d A}{d z}=\frac{a M+\alpha(1-a)}{\beta+c}, \frac{d M}{d z}=P, \quad \frac{d P}{d z}=\frac{d^{2} M}{d z^{2}}=-c P+\frac{\gamma M}{1+M}-a M .
$$

A periodic traveling wave (PTW) solution is a periodic orbit or a limit cycle solution of this ODE system (3). Figure 2 shows the existence of PTW solutions of (1) as a function of parameter $\delta$ and the wave speedc. Here, we consider the parameter $\delta$ as a control parameter while the other parameters are $\alpha=0.6667, \beta=41.5692$ and $\gamma=0.1333$. The range $0.8<\delta<0.2$ has considered. 
The rectangle on the parameter plane indicates that there is no PTW on that point. The values on the parameter plane indicate the periods of the PTW solutions of a given point of the wave speed $c$ and the control parameter $\delta$. At this stage, we do not find whether the waves in the parameter plane are stable or unstable. Later, we will investigate the stability information of the waves.

Figure 3 indicates two PTWs of (3) as a function of the parameter $\delta$ and the wave speed $c$. These Figures indicate that, increasing the value of control parameter $\delta$ increases the periods of the PTW solutions. However, the periods are not changing quickly as $\delta$ changes. The other parameter values are the same as in fig. 2.

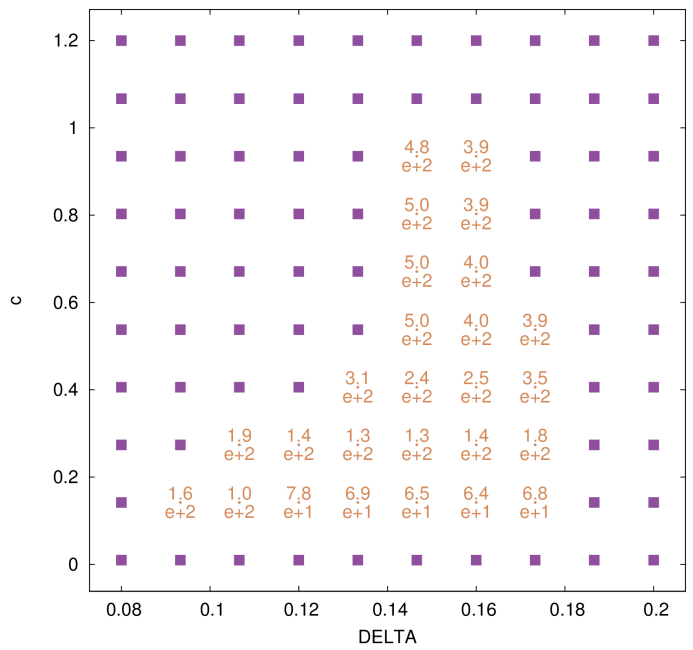

Fig. 2. The existence of PTW solutions of (1) as a function of the parameter $\delta$ and the wave speed c for $\alpha=$ $0.6667, \beta=41.5692$ and $\gamma=0.1333$. The symbols and values show the results on the $10 \times 10$ grid in the parameter plane: small filled rectangle indicates that there is no PTW exist at that point. The values on the parameter plane indicate the periods of the PTW solutions of a given point of $\delta$ and $\mathrm{c}$.
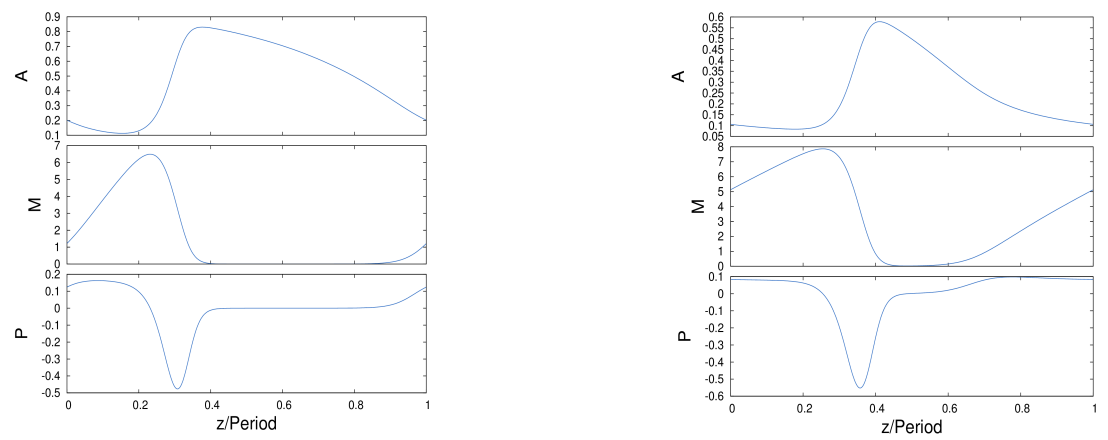

Fig. 3. Two periodic traveling wave solutions of (3) as a function of the parameter $\beta$ and the wave speed c. A, M, Prepresent the internal names of state variables; refer to [15]. The other parameter values are $\alpha=0.6667$, $\beta=41.5692$ and $\gamma=0.1333$. (a) The first one is for the parameter $\delta=0.16$ and the wave speedc $=0.30$. The period of the wave is 154.470 . (b) The second one is for the parameter $\delta=0.12$ and the wave speed $\mathrm{c}=0.30$. In this case the period of the wave is 168.072. 
From the ecological point of view, we consider $\delta$ as a key control which reflects the algal concentrations in the upper water layers. An important step in calculating the parameterregiongiving patterns is to set up bifurcation diagram for (1) as $\delta$ is varied with fixed wave speed $c$. We use the steady state (2) as a starting point of bifurcation diagram since it is stable to homogeneous perturbations. An example of bifurcation diagramis shown in fig. 4. At large values of $\delta$ there are no patterns, but as $\delta$ is decreased there is a Hopf bifurcation in which a branch of limit cycle (pattern) solutions emanates. This solution branch terminates at a homoclinic point, meaning that the period of the limit cycle tends to infinity; thus as bifurcation parameter $\delta$ is decreased towards this point the mussels bands become more and more widely separated. For values of the wave speed $c$ close to that used in fig. 1, it is the loci of the Hopf bifurcation and homoclinic point that enclose the region of the $\delta-c$ parameter plane giving patterns.

The simple form of the bifurcation diagram in Figure 4 does not apply for all values of c: two complications can arise. First, the branch of pattern solutions may have a fold, rather than proceeding monotonically from the Hopf bifurcation to the homoclinic point (e.g. Figure 4). In some parts of the $\delta$-c parameter plane the locus of this fold forms one boundary of the parameter region giving patterns. Note that in general, for any system of ODEs, the part of a parameter plane in which limit cycles exist is bounded by segments of three types of loci: Hopf bifurcations, homoclinic points, and folds in a limit cycle solution branch.

\section{Stability of Periodic Traveling Wave Solutions}

In application, we need to investigate not only the existence of PTW solutions but also whether the solutions are stable or unstable in the parameter plane. In order to study the stability of the PTWs, one needs to linearize it via the traveling wave solution. Substituting $a(x, t)=A(z)$ and $m(x, t)=$ $M(z)$ in (1) and neglecting non-linear term yield:

$$
\begin{gathered}
\frac{\partial a_{l i n}}{\partial t}=\frac{\partial a_{l i n}}{\partial x}+a_{l i n}(-\alpha-M)+m_{l i n}(-A) \\
\frac{\partial m_{l i n}}{\partial t}=\frac{\partial^{2} m_{l i n}}{\partial x^{2}}+a_{l i n}(\delta m)+m_{l i n}\left(A \delta-\frac{\gamma}{(1+M)^{2}}\right)
\end{gathered}
$$

where $a_{\text {lin }}(x, t)=a(x, t)-A(z)$ and $m_{\text {lin }}(x, t)=m(x, t)-M(z)$.

Now, substituting $a_{\text {lin }}(x, t)=e^{\lambda t} A_{\text {lin }}(z)$ and $m_{\text {lin }}(x, t)=e^{\lambda t} M_{\text {lin }}(z)$ in (4), we get the following eigenvalue problem:

$$
\begin{gathered}
\lambda A_{\text {lin }}=(\beta+c) \frac{d A_{\text {lin }}}{d x}+A_{\text {lin }}(-\alpha-M)+M_{\text {lin }}(-A), \\
\lambda M_{\text {lin }}=\frac{d^{2} M_{\text {lin }}}{d x^{2}}+c \frac{d M_{\text {lin }}}{d x}+A_{\text {lin }}(\delta m)+M\left(A \delta-\frac{\gamma}{(1+M)^{2}}\right),
\end{gathered}
$$

with the boundary conditions, using Floquet theory,

$$
A_{\text {lin }}(L)=A_{\text {lin }}(0) e^{i \gamma}, M_{\text {lin }}(L)=M_{\text {lin }}(0) e^{i \gamma} \text {,for some } \gamma \in \mathbb{R}
$$


where $L$ is the period of the PTW, $\gamma$ denotes the phase shift across one period of the wave, $\vec{A}_{\text {lin }}$ represents the eigenfunction, and $\lambda$ is the eigenvalue. In order to make the boundary conditions regular and re-scale $L$ to unity, it is required to use a transformation, namely Bloch transformation. Then, one can solve the above problem numerically by using any regular numerical method. Hence, the eigenvalue equation (5) can be reduced to the first order ODE system as follows:

$\frac{d A_{\text {lin }}}{d z}=A_{\text {lin }} \frac{\lambda}{\beta+c}+A_{\text {lin }} \frac{\alpha+M}{\beta+c}+M_{\text {lin }} \frac{A}{\beta+c}, \frac{d M_{\text {lin }}}{d z}=P_{\text {lin }}$,
$\frac{d P_{\text {lin }}}{d z}=\frac{d^{2} M_{\text {lin }}}{d z^{2}}=\lambda M_{\text {lin }}-c P_{\text {lin }}-A_{\text {lin }}(\delta m)-M_{\text {lin }}\left(A \delta-\frac{\gamma}{(1+M)^{2}}\right)$.

This implies,

$$
\frac{d}{d z}\left[\begin{array}{c}
A_{\text {lin }} \\
M_{\text {lin }} \\
P_{\text {lin }}
\end{array}\right]=(C(z)+\lambda D)\left[\begin{array}{c}
A_{\text {lin }} \\
M_{\text {lin }} \\
P_{\text {lin }}
\end{array}\right],
$$

where $C$ and $D$ are the $3 \times 3$ matrices given by

$$
\begin{gathered}
C=\left[\begin{array}{ccc}
\frac{\alpha+M}{\beta+c} & \frac{A}{\beta+c} & 0 \\
0 & 0 & 1 \\
-\delta M & \frac{\gamma}{(1+M)^{2}}-\delta A & -c
\end{array}\right] \\
D=\left[\begin{array}{ccc}
\frac{1}{\beta+c} & 0 & 0 \\
0 & 0 & 0 \\
0 & 1 & 0
\end{array}\right]
\end{gathered}
$$

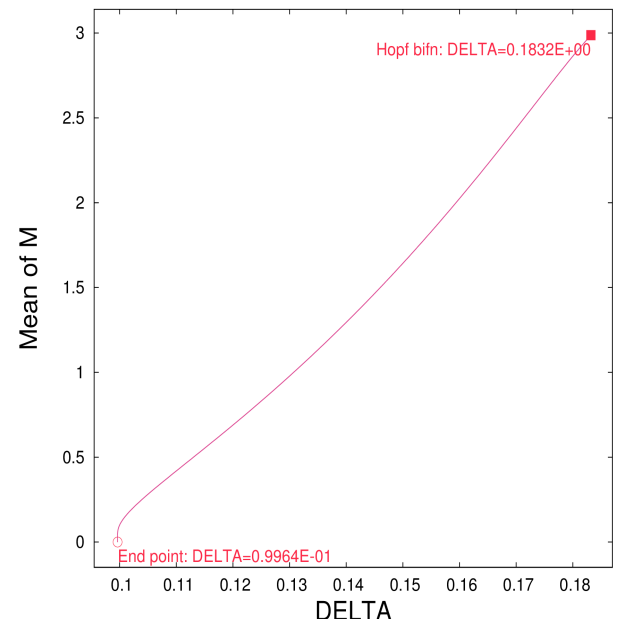

Fig. 4. Bifurcation diagrams for (1) showing the mean of mussel density as a functions of $\delta$ with wave speed $\mathrm{c}=0.1$. The other parameter are $\alpha=0.6667, \beta=41.5692$ and $\gamma=0.1333$. The square indicates a Hopf bifurcation point and open circle indicates a homoclinic point. 


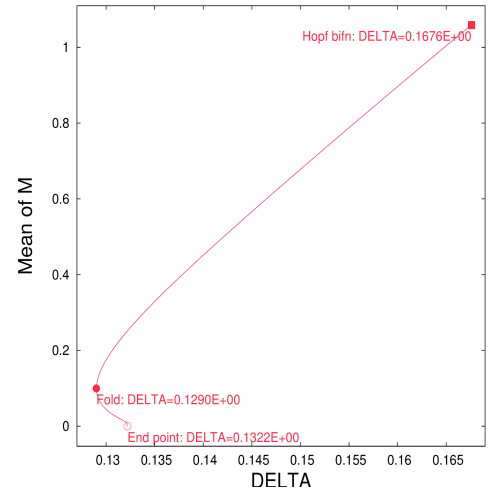

Fig. 5. Bifurcation diagrams for (1) showing the mean of mussel density as a functions of $\delta$ with wave speed $c=0.05$. The values of other parameters arethe same as in fig. 4. The square indicates a Hopf bifurcation point, open circle indicates a homoclinic point and closed circle is a fold in the solution branch.

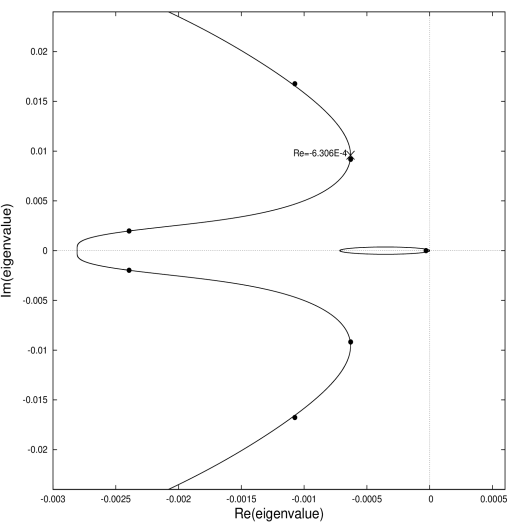

(a) $\mathrm{c}=0.48$

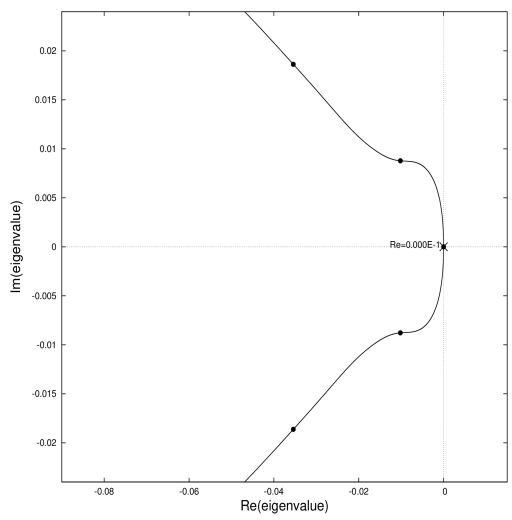

(c) $\mathrm{c}=0.15$

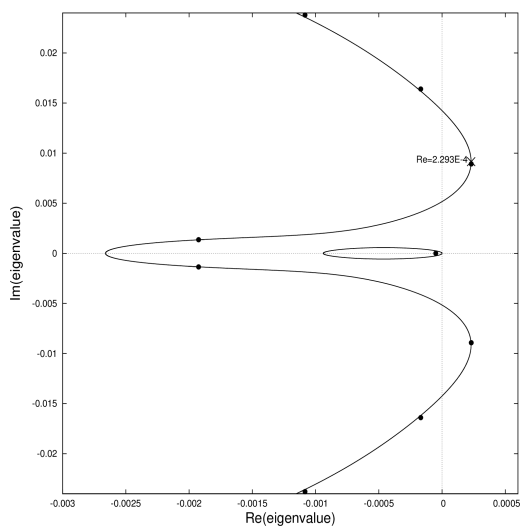

(b) $\mathrm{c}=0.485$

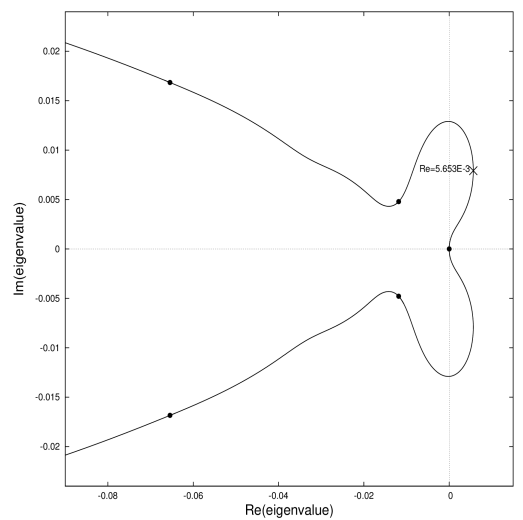

(d) $\mathrm{c}=0.08$

Fig. 6. Eigenvalue spectra for PTWs of the model (1), for $\delta=0.16$ and (a) $c=0.48$; (b) $c=0.485$; (c) $c=0.15$; (d) $\mathrm{c}=0.08$. In (a) and (c) the spectrum extends into the left hand half plane, so that pattern is stable; in (b) and (d) the pattern is unstable. Stability of Hopf type occurs inc $\in(0.48,0.485)$; the change in stability is of Eckhaus type for $\mathrm{c} \in(0.08,0.15)$. The other parameters are $\alpha=0.6667, \beta=41.5692$ and $\gamma=0.1333$. 
The spectrum of the PTW is a non-trivial solution of (5) and (6) for the set of eigenvalues $\lambda$. For $\gamma=0$, one can calculate solutions of (5) and (6) using standard numerical method. However, when $\gamma \neq 0$, one cannot reduce (5) and (6) to form in matrix eigenvalue problem and hence this can be calculated by numerical continuation method for the different phase of $\gamma$. To perform a continuous numerical calculation in the phase difference $\gamma=0$, one might calculate matrix eigenvalues and eigenfunctions as initial data. This calculation must be used over $0<\gamma<2 \pi$ for each of the designated matrix eigenvalues with largest real part. More continuation procedure of these implementations requires for some special settings including set orthogonality conditions, ensuring the normalization of integral constraints in the eigenfunctions etc.; full details are given in [12].

To understand the stability of PTW solution, we need to calculate the essential spectra of the PTW solutions. Generally there are two parts; "discrete part" and "continuous part", in the spectra of linear operators and those parts are respectively known as point spectrum and essential spectrum [11]. However the point spectrum (discrete part) is empty for the PTWs, and thus, the spectrum of the PTWs consists of the essential spectrum (continuous part) [15]. Moreover, a spectrum of a PTW solution in a large domain is the continuous spectrum [12], [17]. A PTWsolution said to be unstable when the curvature of spectrum crosses the imaginary axis; otherwise, it is stable. Generally stability change of the PTWs of partial differential equations (PDEs) has two types: Eckhaus type and Hopf type. A change of stability is said to be Eckhaus (sideband) type when the spectrum of the solution changes sign at the origin. However, if a fold is generated in the curvature of the spectrum and crosses the imaginary axis away from the origin is known as a stability change of Hopf type [22], [23].

In fig. 6, we show the essential spectra of the PTWs of model (1). Plot 6(a) represents an essential spectrum for a stable PTW for $\delta=0.155$ and $c=0.48$ since the spectrum is extended into the left half of the complex plane. Plot 6(b) shows an essential spectrum for an unstable PTW for $\delta=$ 0.155 and $c=0.485$ since the spectrum crossed the imaginary axis. Both of these figures have a fold, which passes through the imaginary away from the origin, meaning that change in stability is Hopf type. On the other hand, In fig. 6(c), essential spectrum locates in the left hand half plane for $\delta=0.155$ and $c=0.15$, so that the pattern is stable. In fig. $6(d)$, an essential spectrum for a unstable PTW for $\delta=0.155$ and $c=0.08$, meaning that the spectrum crosses the imaginary axis. The change in stability inc $\in(0.08,0.15)$ is Eckhaus type, meaning that it occurs via a change in the sign of the curvature of the spectrum at the origin.

\section{Conclusion}

Regular spatial pattern formation is a central feature of natural phenomenon in a wide range of ecosystems. Recent evidence shows that spatial patterns become an important indicator for catastrophic regime shifts as a result of changing conditions, e.g., climate change. Therefore, it is important to investigate the mechanism of pattern formation in the young mussel beds. Numerical 
bifurcation analysis is a major way to understand the mechanism of patterned solutions of PDE models. Therefore, in this paper, we have considered this approach to study van de Koppel model (1) for the banded pattern formation in soft-bottomed mussels beds. We have unraveled the complicated dependence on parameter values of pattern existence. The most significant aspect of our results was the calculation of the region of pattern existence and the stability of the solutions. We have found that the periodic solution pattern born at Hopf point and terminates at homoclinic bifurcation point. The detailed stability analysis of the PTWs at the different grids we will consider for our next paper.

\section{REFERENCES}

[1] Camazine, S., 2003. Self-organization in biological systems.Princeton University Press.

[2] De Waal, F. B. and Tyack, P. L., 2009. Animal social complexity: intelligence, culture, and individualized societies. Harvard University Press.

[3] Gascoigne, J. C.; Beadman, H. A.; Saurel, C. and Kaiser, M. J. (2005). Density dependence, spatial scale and patterning in sessile biota, Oecologia $145: 371-381$.

[4] Klausmeier, C. A. (1999). Regular and irregular patterns in semiarid vegetation, Science 284 : 18261828.

[5] Koppel, J. v. d. and Crain, C. M. (2006). Scale-dependent inhibition drives regular tussock spacing in a freshwater marsh, The American Naturalist 168 : E136-E147.

[6] Koppel, J. v. d.; Rietkerk, M.; Dankers, N. and Herman, P. M. (2005). Scale-dependent feedback and regular spatial patterns in young mussel beds, The American Naturalist 165 : E66-E77.

[7] Lejeune, O.; Tlidi, M. and Couteron, P. (2002). Localized vegetation patches: a self-organized response to resource scarcity, Physical Review E 66 : 010901.

[8] Levin, S. A. (1992). The problem of pattern and scale in ecology: the Robert H. MacArthur award lecture, Ecology 73 : 1943-1967.

[9] Liu, Q.-X.; Herman, P. M.; Mooij, W. M.; Huisman, J.; Scheffer, M.; Olff, H. and Van De Koppel, J. (2014). Pattern formation at multiple spatial scales drives the resilience of mussel bed ecosystems, Nature communications $5: 5234$.

[10] Parrish, J. K. and Edelstein-Keshet, L. (1999). Complexity, pattern, and evolutionary trade-offs in animal aggregation, Science 284 : 99-101.

[11] Rademacher, J. D.; Sandstede, B. and Scheel, A. (2007). Computing absolute and essential spectra using continuation, Physica D: Nonlinear Phenomena 229 : 166-183.

[12] Rademacher, J. D. and Scheel, A. (2007). Instabilities of wave trains and Turing patterns in large domains, International Journal of Bifurcation and Chaos $17: 2679-2691$.

[13] Rietkerk, M. and Van de Koppel, J. (2008). Regular pattern formation in real ecosystems, Trends in ecology \& evolution 23 : 169-175.

[14] Ritchie, M. E. and Olff, H. (1999). Spatial scaling laws yield a synthetic theory of biodiversity, Nature 400 : 557.

[15] Sherratt, J. A. (2012). Numerical continuation methods for studying periodic travelling wave (wavetrain) solutions of partial differential equations, Applied Mathematics and Computation 218 : 4684-4694.

[16] Sherratt, J. A. (2013). History-dependent patterns of whole ecosystems, Ecological Complexity 14 : 8-20.

[17] Sherratt, J. A. (2013). Numerical continuation of boundaries in parameter space between stable and unstable periodic travelling wave (wavetrain) solutions of partial differential equations, Advances in Computational Mathematics $39: 175-192$. 
[18] Solé, R. V. and Bascompte, J., 2006. Self-Organization in Complex Ecosystems. (MPB-42). Princeton University Press.

[19] Viswanathan, G. M.; Da Luz, M. G.; Raposo, E. P. and Stanley, H. E., 2011. The physics of foraging: an introduction to random searches and biological encounters. Cambridge University Press.

[20] Wang, R.-H.; Liu, Q.-X.; Sun, G.-Q.; Jin, Z. and van de Koppel, J. (2009). Nonlinear dynamic and pattern bifurcations in a model for spatial patterns in young mussel beds, Journal of the Royal Society Interface 6 : 705-718.

[21] Weerman, E. J.; Van de Koppel, J.; Eppinga, M. B.; Montserrat, F.; Liu, Q.-X. and Herman, P. M. (2010). Spatial self-organization on intertidal mudflats through biophysical stress divergence, The American Naturalist 176 : E15-E32.

[22] Gani, M. O., \& Ogawa, T. (2015). Instability of periodic traveling wave solutions in a modified FitzHugh-Nagumo model for excitable media. Applied Mathematics and Computation, 256, 968-984.

[23] Gani, M. O., \& Ogawa, T. (2016). Stability of periodic traveling waves in the Aliev-Panfilov reactiondiffusion system. Communications in Nonlinear Science and Numerical Simulation, 33, 30-42. 